\title{
USO DE FUENTES NO CONVENCIONALES DE NITRÓGENO EN LA FERTILIZACIÓN DEL MAÍZ (Zea mays L.), EN CAÑETE (PERÚ). I: RENDIMIENTO Y EXTRACCIÓN DE N, P Y K
}

\section{USE OF NON-CONVENTIONAL SOURCES OF NITROGEN IN CORN (Zea mays L.) FERTILIZATION IN CAÑETE (PERÚ). I: YIELD AND N, P AND K UPTAKE}

\author{
Guillermo Aguirre Yato $^{1}$ y Julio Alegre Orihuela ${ }^{1}$
}

\begin{abstract}
Resumen
La industria cárnica y pesquera produce diversos residuos, que pueden ser usados en la agricultura. Para comparar el uso de harina de cuernos y pezuñas (HCP), y de residuos hidrolizados $(\mathrm{RH})$ de la industria pesquera, con la fertilización mineral, se desarrolló un experimento de campo en el valle de Cañete (Perú), en el cultivo de maíz. Se cuantificaron la producción total de biomasa, el rendimiento de grano y el índice de cosecha (IC), encontrándose diferencias significativas entre tratamientos, correspondiendo los mayores rendimientos de biomasa y grano para NPK, HCP+PK y RH+NPK y menores rendimientos para los tratamientos, RH solo, 0PK, y testigo 000. Los IC variaron entre 0.48 y 0.52 .

Las extracciones promedio, alcanzaron en $\mathrm{kg} \mathrm{t}^{-1}$ de grano a 23.8 de $\mathrm{N}, 12.3$ de $\mathrm{P}_{2} \mathrm{O}_{5}, 23.6$ de $\mathrm{K}_{2} \mathrm{O}$. Las concentraciones de nutrientes siguieron el siguiente orden: grano $>$ panoja $>$ hojas $>$ tuza $>$ tallo $>$ brácteas, para N; grano $>$ panoja $>$ hojas $>$ tallo, brácteas, tuza, para P; tallo $>$ hojas $>$ brácteas, panoja, tuza $>$ grano, para K.

Por consiguiente la HCP puede ser usada como fuente de nitrógeno así como los fertilizantes químicos para sistemas con cultivos, requiriéndose mayores estudios en el uso de residuos hidrolizados.
\end{abstract}

Palabras clave: harina de cuernos y pezuñas, residuo hidrolizado, extracción de nutrientes, concentración de nutrientes.

\begin{abstract}
Meat and fish industry produce residues that can be used in agriculture. With this purpose, an experiment was developed at Cañete Valley (Perú), in a maize crop field, in order to compare the use of hooves and horn meal (HHM), hydrolyzed residues (HR) from the fish industry and chemical fertilization. Total biomass production, grain yield and harvesting index were quantified. There were significant differences among treatments. The highest biomass production and grain yields were found in the NPK, HHM + PK and HR + NPK treatments. Treatments with HR, 0PK, 000 presented the lowest production. Harvesting indexes ranged between $0.48-0.52$.

On average, nutrient extraction in $\left(\mathrm{kg} \mathrm{t}^{-1}\right)$ of grain yield reached the following results: $23.8 \mathrm{~N}$, $12.3 \mathrm{P}_{2} \mathrm{O}_{5}, 23.6 \mathrm{~K}_{2} \mathrm{O}$. Nitrogen concentration in parts of the plant had the following order: grain $>$ corn tassel $>$ leaves $>\mathrm{cob}>$ stalk $>$ cob husk, for nitrogen. For phosphorus it was as follows: grain $>$ corn tassel $>$ leaves $>$ stalk $>$ cob husk and cob. For potassium: stalk $>$ leaves $>$ cob husk and corn tassel $>$ cob $>$ grain.

In conclusion, the HHF can be used as a source of nitrogen in the same way as chemical fertilizers for cropping systems but more studies are needed in relation to the use of HR.

Key words: hooves and horn meal, hydrolyzed residues, nutrient uptake, nutrient concentration.
\end{abstract}

\section{Introducción.}

La industria de productos cárnicos genera una gran cantidad de desechos orgánicos (ej. cuernos, pezuñas, pelos, huesos, sangre, etc.). Debido a la falta de procesos sanitarios adecuados en varios países de Latinoamérica, estos residuos no son tratados adecuadamente (Garzón, 2010), representando una fuente potencial de contaminación. Sin embargo, su elevado contenido de elementos esenciales como nitrógeno, fósforo (Zoccola et al., 2009), y microelementos, los hacen muy interesantes para su uso en agricultura en forma de fertilizantes no convencionales. Su uso puede ser empleado en especial en pequeñas huertas familiares o agricultura 
de pequeña escala, reduciendo el uso de fertilizantes químicos.

Diversas experiencias indican que estos materiales pueden ser usados también como enmiendas orgánicas, mejorando las propiedades del suelo. La combinación de composta de lombriz y harina de huesos incrementó el carbono orgánico total, carbono orgánico particulado, densidad aparente, fósforo extractable, $\mathrm{pH}$, conductividad eléctrica y CIC del suelo (Comese et al., 2009). Así mismo, es importante destacar el uso de cuernos y pezuñas como fertilizantes a nivel comercial por parte de las industrias, a partir de lo cual surge la idea de su uso a nivel más doméstico sin problemas adicionales.

Cuando se cuantifica la producción de residuos en los camales de las grandes urbes resultan volúmenes considerables, lo que ha llevado a los estudiosos a considerar su uso en la agricultura (López \& Sainz, 2011).

En la mayoría de investigaciones sobre el uso de residuos sólidos, se apuesta por procesos de digestión anaerobia y/o composta en lugar de la aplicación directa. Por ejemplo, Xia et al. (2015) realizó la digestión anaerobia de las harinas de pezuñas con fines de producción gasífera. Por otro lado, Cayuela et al. (2009), realizó la composta con harina de cuernos y pezuñas.

Utilización de los residuos de pescado.

La producción de residuos en la industria pesquera (ej. vísceras, pieles, huesos, sangre, cabezas, colas y aletas), puede alcanzar entre 20 al $70 \%$ de la materia prima original (Vincenza et al., 2010). Se estima que en el mundo se generan alrededor de 20 millones de toneladas anuales de residuos pesqueros (Windsor \& Barlow, 1984). Considerando un contenido proteico de entre 15 a $20 \%$, éstos representan aproximadamente 3 a 4 millones de toneladas de proteínas. Gran parte de estos residuos se utilizan en la alimentación animal, así como en la elaboración de fertilizantes (Bossio, 2007). Su composición de proteínas explica de por sí su alto valor nutricional. Los residuos hidrolizados.

El hidrolizado se produce mediante la molienda de desechos de pescado, la adición de un ácido y luego la agitación de la mezcla hasta que el proceso de hidrólisis enzimática esté completo (Knuckey et al., 2004). El ácido sulfúrico es utilizado debido a su fácil disponibilidad y manejo, bajo costo y contenido de azufre, que beneficia a los cultivos. El ácido sulfúrico adicionado ( $2 \%$ en peso) ayuda a dar inicio a la hidrólisis de pescado, pero su objetivo principal es reducir el $\mathrm{pH}$ para prevenir el ataque de otros microorganismos.

Bossio (2007), cita a Aspmo et al. (2005), indicando que produjeron hidrolizados de vísceras de pescado utilizando enzimas endógenas y enzimas exógenas comerciales como papaína y bromelina de origen vegetal, y alcalasa de origen microbiano.
Además encontraron que la alcalasa proporciona la mayor solubilización de materia seca. Estos hidrolizados son también una fuente excelente de nitrógeno para el cultivo de bacterias ácidolácticas.

Producción de biomasa, rendimientos y extracción de nutrientes por el cultivo de maíz en condiciones de campo.

Los análisis de suelos y la cuantificación de la extracción de nutrientes son una herramienta para una adecuada recomendación de fertilización. En el caso del maíz, se han encontrado cifras distintas pero referenciales, que están en función de factores como son las, variedades, la época de siembra, la expectativa de rendimiento y la localidad (Vásquez et al., 2014).

Arrieche \& Mora (2005), usando tres fuentes de materia orgánica (estiércol de pollo, estiércol de pollo compostado con enzimas, y cachaza de caña de azúcar compostada con enzimas), encontraron efectos de incremento de la materia orgánica en el suelo, así como incrementos en la concentración de nitrógeno en las hojas, y en el rendimiento de grano; comparadas con un testigo sin uso de materia orgánica.

En experimentos en dos localidades de la Provincia de Bolívar (Ecuador), aplicando la metodología del elemento faltante, se encontraron diferencias significativas para la producción de grano y no para la tuza y residuos. En cuanto a la concentración y extracción de nutrientes las mayores diferencias que se encontraron fueron para el nitrógeno (Yanez et al., 2010).

Silva et al. (2009), al evaluar las características morfológicas, agronómicas y la producción en diferentes fechas de siembra del maíz, concluyen que son más dependientes de la época de siembra, que de la carga genética, por la oportunidad de la época de floración de las plantas.

Prystupa et al. (2004), en ensayos de niveles de P y $\mathrm{S}$, en combinación con y sin aplicación de $\mathrm{K}$, en siete lugares, no encontraron efectos del K, en cambio sí se encontró diferencias con las dosis de $\mathrm{P}$ y S.

Setiyono et al. (2010), consideran que para la estimación de los requerimientos nutricionales de los cultivos, se pueden usar modelos de simulación como el QUEFTS (Quantitative Evaluation of the Fertility of Tropical Soils), con el que establecieron límites máximos y mínimos por Índices de Eficiencia (IE), encontrando en $\mathrm{kg}$ de grano por $\mathrm{kg}$ de nutriente en la materia seca, para rendimientos de $20 \mathrm{Mg} \mathrm{ha}^{-1}, 40$ a 83 $\mathrm{kg}$ de grano $\mathrm{kg}^{-1} \mathrm{~N}, 225$ a $726 \mathrm{~kg}$ grano $\mathrm{kg}^{-1} \mathrm{P}$ y 29 a $125 \mathrm{~kg}_{\text {grano }} \mathrm{kg}^{-1} \mathrm{~K}$. Por tanto, el modelo predijo un incremento lineal en rendimiento de grano, de $16.4 \mathrm{~kg}$ $\mathrm{N}, 2.3 \mathrm{~kg}$ P y $15.9 \mathrm{~kg} \mathrm{~K}$ por cada tonelada de grano, para alcanzar $60-70 \%$ del rendimiento potencial.

$\mathrm{Xu}$ et al. (2013) y Setiyono et al. (2010), haciendo uso del modelo QUEFTS, establecieron relaciones óptimas de N: P: K de $4.83: 1: 4.37$ para la biomasa de plantas de maíz en primavera, y de $4.61: 1: 3.61$ para maíz en verano. 
Hu et al. (2010), analizaron el efecto de la aplicación de fertilizantes en la producción de biomasa, conjugando tratamientos con la metodología del elemento excluido, para determinar la distribución de los elementos N, P, K, Cu, Zn, Fe y Mn, de varios órganos del maíz, concluyendo que el contenido de $\mathrm{N}$ siguen el orden de hojas $>$ grano $>$ tallo; para el $\mathrm{P}$ grano $>$ hojas $>$ tallos; para el $\mathrm{K}$ fue tallo $>$ hojas $>$ grano, y para el Fe el contenido fue hojas $>$ tallo $>$ grano. Para el caso del $\mathrm{Cu}$ y $\mathrm{Zn}$ los datos fueron similares. Respecto a los rendimientos de biomasa, el tratamiento NPK fue mayor que NP, NK y PK.

La viabilidad del uso de aguas servidas y de residuos compostados, en combinación con fertilizantes sintéticos para la producción de biomasa en maíz fue ensayada a nivel de campo (Seleiman et al., 2013), obteniendo hasta $30 \mathrm{t} / \mathrm{ha}$ de biomasa con un contenido promedio de $1.4 \%$ de $\mathrm{N}$ en las hojas. Los autores concluyen que el uso de residuos no convencionales permite mejorar la sostenibilidad de la agricultura, sin incrementar el uso de fertilizantes sintéticos.

La extracción unitaria de nutrientes para el maíz ha sido reportada en $27.3 \mathrm{~kg}$ de N, $5.9 \mathrm{~kg}$ de $\mathrm{P}$ y $29.1 \mathrm{~kg}$ de K. por tonelada de grano (Vásquez et al., 2014).

En el presente trabajo se propone que la harina de cuernos y pezuñas, y el residuo hidrolizado de pescado son fuentes que proveen un adecuado suministro de nitrógeno para el cultivo de maíz en suelos arenosos de la costa central del Perú.

Los objetivos del presente trabajo son, determinar la viabilidad del uso de los residuos hidrolizados, y de otros materiales como harina de cuernos y pezuñas, desechos básicamente orgánicos, bajo condiciones de campo y cuantificar la extracción de nutrientes.

\section{Materiales y métodos.}

Caracterización físico-química de los materiales orgánicos utilizados en el experimento.

La harina de cuernos y pezuñas (HCP) fue obtenida a partir de una empresa local de acuerdo al proceso descrito por Documet (2015). En breve: los cuernos y pezuñas fueron hervidos en agua durante 10 minutos, enfriados (para desprender la clavija ósea de los cuernos), y posteriormente secados en horno. El material seco fue molido en molino de martillos y tamizado hasta un tamaño de partícula que pase por malla 16 Mesh (aprox. $1.2 \mathrm{~mm}$ de diámetro).

El residuo hidrolizado de pescado (RHP) fue provisto por el Laboratorio de Bioremediación del Departamento de Biología (Facultad de Ciencias UNALM). Este producto fue obtenido mediante la degradación enzimática de residuos frescos de pescado mediante bacterias acidolácticas (Bossio, 2007). Durante este proceso, se pueden obtener aminoácidos y péptidos de variados tamaños (Rustad, 2003).
El análisis químico de los residuos es resumido en la Tabla 1. La HCP tiene un elevado contenido de N además de pequeñas cantidades de los demás elementos, presentando un elevado potencial como fertilizante (Korniłłowicz \& Bohacz, 2011). Debido a que la queratina que la constituye es degradada lentamente por hongos queratinofílicos en el suelo, es una fuente de liberación de nitrógeno permanente pero lenta (Zoccola et al., 2009). El RHP es rico en nitrógeno y potasio, y su disponibilidad es mayor. Ubicación y descripción del experimento.

El experimento fue instalado en un campo agrícola del fundo "San Martín" del Instituto Regional de Desarrollo - Costa de la UNALM. El análisis de la capa arable $(20 \mathrm{~cm})$ indica un suelo de textura franco

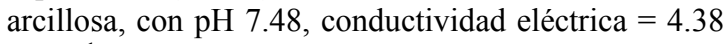
$\mathrm{dS} \mathrm{m}{ }^{-1}$, M.O. $=1.82 \%$, $\mathrm{P}$ disponible $($ Olsen $)=21.2$ $\mathrm{mg} \mathrm{kg}^{-1}, \mathrm{~K}$ disponible (acetato de amonio) $=286 \mathrm{mg}$ $\mathrm{kg}^{-1}$, y CIC $=15.2 \mathrm{cmol}_{\mathrm{c}} \mathrm{kg}^{-1}$.

Maíz (Zea mays) var. "Santa Elena 5070", fue sembrado a un distanciamiento de $0.90 \mathrm{~m}$ entre surcos y $0.40 \mathrm{~m}$ entre golpes, con 2 y 3 semillas por golpe alternado (69 400 plantas/ha). Parcelas de cuatro surcos de $6 \mathrm{~m}$. de largo fueron seleccionadas como unidades experimentales. Seis tratamientos fueron aplicados: T1: fertilización completa 200-100-70 empleando urea $(46 \% \mathrm{~N}), \mathrm{T} 2$ : fertilización completa empleando HCP, T3: RHP (50 1/ha), T4: fertilización completa más RHP, T5: fertilización sin nitrógeno 0100-70 y, T6: control absoluto 0-0-0. El nitrógeno fue fraccionado en dos momentos, a los 40 y 70 días después de la siembra. Superfosfato triple de calcio $\left(46 \% \mathrm{P}_{2} \mathrm{O}_{5}\right)$ y cloruro de potasio $\left(60 \% \mathrm{~K}_{2} \mathrm{O}\right)$ fueron empleados como fuentes de fósforo $\mathrm{y}$ potasio, respectivamente; y fueron aplicados al primer cultivo, junto con la primera aplicación de nitrógeno. Los fertilizantes sólidos fueron aplicados en puyados, mientras que el RHP líquido fue aplicado vía foliar. Se instalaron cuatro repeticiones para cada tratamiento. Los riegos, cultivos y el control de plagas fueron manejados oportunamente.

$\underline{\text { Cosecha y muestreo. }}$

Al momento de cosecha, se seleccionó un área de evaluación consistente de los dos surcos centrales de cada parcela, a fin de descartar el efecto de borde. La altura de planta fue medida en diez plantas al azar. El rendimiento fue calculado a partir de la producción de

Tabla 1. Composición química de la harina de cuernos y pezuñas y residuo hidrolizado de pescado.

\begin{tabular}{lcc}
\hline & $\begin{array}{c}\text { Harina de cuernos } \\
\text { y pezuñas }\end{array}$ & Residuo Hidrolizado \\
\hline $\mathrm{pH}$ & 6.20 & 3.6 \\
$\mathrm{CE}(\mathrm{dS} / \mathrm{m})$ & 10.90 & 17.5 \\
Materia & $96.52 \%$ & $103.2 \mathrm{~g} / 1$ \\
orgánica & & \\
$\mathrm{N}$ & $13.58 \%$ & $1876 \mathrm{mg} / 1$ \\
$\mathrm{P}_{2} \mathrm{O}_{5}$ & $0.16 \%$ & $167 \mathrm{mg} / 1$ \\
$\mathrm{~K}_{2} \mathrm{O}$ & $0.32 \%$ & $1890 \mathrm{mg} / 1$ \\
\hline
\end{tabular}


Tabla 2. Comparación de medias por la prueba de Bonferroni $(\alpha=0.05)$ para las distintas variables en las modalidades de fertilización en el cultivo de maíz en la localidad de San Martín (Valle de Cañete).

\begin{tabular}{lcccccc}
\hline Tratamiento & $\begin{array}{c}\text { Alt.planta } \\
\text { (cm) }\end{array}$ & $\begin{array}{c}\text { Biomasa Total } \\
\text { (kg/ha) }\end{array}$ & $\begin{array}{c}\text { Rendimiento } \\
\text { (kg/ha) }\end{array}$ & $\begin{array}{c}\text { Extracc. } \mathbf{c} \\
\text { (kg/ha) }\end{array}$ & $\begin{array}{c}\text { Extrac. P } \\
\text { (kg/ha) }\end{array}$ & $\begin{array}{c}\text { Extracc. K } \\
\text { (kg/ha) }\end{array}$ \\
\hline NPK min. & $241 \mathrm{a} *$ & $19386 \mathrm{a}^{*}$ & $10231 \mathrm{a}^{*}$ & $242 \mathrm{a}^{*}$ & $63 \mathrm{a}^{*}$ & $192 \mathrm{ab}^{*}$ \\
HCP + PK & $241 \mathrm{a}$ & $19579 \mathrm{a}$ & $10271 \mathrm{a}$ & $247 \mathrm{a}$ & $53 \mathrm{~b}$ & $183 \mathrm{ab}$ \\
RHP & $232 \mathrm{ab}$ & $15470 \mathrm{~b}$ & $7375 \mathrm{bc}$ & $179 \mathrm{c}$ & $39 \mathrm{c}$ & $174 \mathrm{bc}$ \\
NPK + RHP & $231 \mathrm{ab}$ & $19259 \mathrm{a}$ & $10050 \mathrm{a}$ & $231 \mathrm{a}$ & $55 \mathrm{~b}$ & $196 \mathrm{a}$ \\
0 P K & $229 \mathrm{~b}$ & $15151 \mathrm{~b}$ & $7730 \mathrm{~b}$ & $178 \mathrm{~b}$ & $44 \mathrm{c}$ & $159 \mathrm{c}$ \\
0 0 0 & $225 \mathrm{~b}$ & $13650 \mathrm{c}$ & $6798 \mathrm{c}$ & $164 \mathrm{~d}$ & $30 \mathrm{~d}$ & $129 \mathrm{~d}$ \\
Media & $\mathbf{2 3 3}$ & $\mathbf{1 7 0 8 3}$ & $\mathbf{8 7 4 3}$ & $\mathbf{2 0 6 . 8}$ & $\mathbf{4 7 . 3}$ & $\mathbf{1 7 2 . 2}$ \\
Mínima & 225 & 13650 & 6798 & 178 & 30 & 129 \\
Máxima & 241 & 19579 & 10271 & 247 & 63 & 192 \\
Desv. estándar & 6.2 & 2557 & 1543 & 33.8 & 10.9 & 22.8 \\
\hline * Promedios que en cada columna comparten una misma letra, no son significativamente diferentes de acuerdo a la \\
\multicolumn{7}{l}{ Prueba de Bonferroni (al 5\% de probabilidades). }
\end{tabular}

grano total de las mazorcas cosechadas. Para obtener la biomasa total, todas las plantas del área de evaluación fueron cortadas a nivel del suelo y pesadas. Cinco plantas de cada parcela fueron separadas en sus órganos: panoja o espiga, hojas, tallo, grano, tuza y brácteas de la mazorca, y pesadas por separado.

Los análisis químicos fueron realizados en el Laboratorio e Invernadero de Fertilidad del Suelo de la UNALM. Los tejidos fueron llevados a estufa a 75 ${ }^{\circ} \mathrm{C}$ para determinar el contenido de materia seca. Muestras de material seco fueron molidas finamente para el análisis químico. El nitrógeno fue determinado por el método de Kjeldahl (Jones, 1991). Porciones de tejidos fueron a sometidas a digestión húmeda con solución nitro-perclórica (Zasoski \& Burau, 1977). El fósforo fue medido por colorimetría, mientras que el potasio fue determinado mediante espectrofotometría de absorción atómica.

La concentración de nutrientes en los diferentes órganos fue empleada para calcular la extracción total de nutrientes por hectárea y extracción por tonelada de grano producido. El índice de cosecha (IC) fue calculado como la relación entre el rendimiento de grano entre la biomasa total.

Análisis estadístico.

Un diseño en bloques completamente al azar
(DBCA) con cuatro repeticiones fue empleado para el experimento. Luego de verificar el cumplimiento de los supuestos de homogeneidad de variancias y normalidad de errores en cada variable, se realizó el análisis de varianza. Para la comparación de medias de los tratamientos, se empleó la prueba de Bonferroni. Los análisis estadísticos se realizaron con la ayuda del paquete Minitab versión 17.

\section{Resultados y discusión.}

Altura de planta, producción de biomasa y rendimiento de grano.

Tanto la altura de planta como la producción de biomasa, representada en forma de peso de materia seca, pueden ser indicadores utilizados en la evaluación de la eficacia de tratamientos (Serna et al., 2011; Ospina et al., 2011). En el presente experimento, se observan dos grupos claramente definidos: los que recibieron fertilización completa NPK, resaltando la HCP y la urea; y por el otro lado, los controles absoluto y sin nitrógeno. La aplicación de RHP mostró escaso efecto (Tabla 2); lo cual puede explicarse por el bajo aporte de nutrientes debido a la dosis aplicada $\left(50 \mathrm{~L} \mathrm{ha}^{-1}\right)$.

Esta misma tendencia fue observada en los rendimientos totales de biomasa y de grano (Tabla 3 ). El IC calculado varió entre 0.48 y 0.53 . Estos valores

Tabla 3. Rendimiento de biomasa, grano (kg/ha), y extracciones unitarias en maíz para distintos tratamientos en San Martín - Cañete.

\begin{tabular}{|c|c|c|c|c|c|c|c|}
\hline \multirow[t]{2}{*}{ Tratamiento } & \multirow{2}{*}{$\begin{array}{c}\text { M.S. } \\
\text { Rastrojo }\end{array}$} & \multirow{2}{*}{$\begin{array}{c}\text { M.S. } \\
\text { grano }\end{array}$} & \multirow{2}{*}{$\begin{array}{c}\text { Total } \\
\text { Biomasa }\end{array}$} & \multirow[t]{2}{*}{ IC } & \multicolumn{3}{|c|}{ Extracción kg t $\mathbf{~ t e ~ g r a n o ~}^{-1}$} \\
\hline & & & & & $\mathbf{N}$ & $\mathbf{P}_{2} \mathbf{O}_{5}$ & $\mathrm{~K}_{2} \mathrm{O}$ \\
\hline NPK min. & $9155 \mathrm{a}^{*}$ & $10231 \mathrm{a}$ & $19386 \mathrm{a}$ & 0.53 & 23.8 & 14.2 & 22.5 \\
\hline $\mathrm{HCP}+\mathrm{PK}$ & $9308 \mathrm{a}$ & $10271 \mathrm{a}$ & $19579 \mathrm{a}$ & 0.52 & 24.1 & 11.8 & 21.4 \\
\hline RH solo & $8095 \mathrm{~b}$ & $7375 \mathrm{bc}$ & $15470 \mathrm{~b}$ & 0.48 & 24.3 & 12.2 & 28.3 \\
\hline $\mathrm{NPK}+\mathrm{RH}$ & 9209 a & $10050 \mathrm{a}$ & 19259 a & 0.52 & 23.1 & 12.7 & 23.4 \\
\hline $0 \mathrm{P} \mathrm{K}$ & $7422 \mathrm{~b}$ & $7730 \mathrm{~b}$ & $15151 \mathrm{~b}$ & 0.51 & 23.3 & 13.1 & 24.7 \\
\hline 000 & $6852 \mathrm{c}$ & $6798 \mathrm{c}$ & $13650 \mathrm{c}$ & 0.50 & 24.4 & 10.2 & 22.8 \\
\hline Media & 8340 & 8743 & 17083 & 0.51 & 23.8 & 12.4 & 23.9 \\
\hline Mínima & 6852 & 6798 & 13650 & 0.48 & 23.1 & 10.2 & 21.4 \\
\hline Máxima & 9308 & 10271 & 19579 & 0.53 & 24.4 & 14.2 & 28.3 \\
\hline Desv. estándar & 1036 & 1543 & 2557 & 0.02 & 0.53 & 1.46 & 2.62 \\
\hline
\end{tabular}


son ligeramente superiores a los encontrados por Vásquez et al. (2014), puede interpretarse y justificarse, ya que la contribución de las raíces a la biomasa no fue considerada.

Los rendimientos de grano (Tabla 3) mostraron un rango de $6798 \mathrm{~kg} \mathrm{ha}^{-1}$ a $10271 \mathrm{~kg} \mathrm{ha}^{-1}$, el cual es esperado para esta variedad y coincide con los obtenidos por otros autores para el cultivo del maíz (Yanez et al., 2010; Delgado et al., 2004; Prystupa et al., 2004; Xu et al., 2013).

Cabe destacar que el desempeño dela HCP como fuente de nitrógeno (Tablas 2 y 3), que fue significativamente similar a la urea, tanto en producción de biomasa como en rendimiento de grano. La aplicación foliar del RHP no mostró efectos favorables, ni cuando fue aplicado como complemento a la fertilización completa ni cuando fue aplicado solo, mostrando resultados semejantes a los controles absoluto y sin nitrógeno.

Extracción de nutrientes (N, P, K).

La extracción de nutrientes mostró la misma tendencia que los rendimientos totales (Tablas 2 y 3 ). $\mathrm{Al}$ ser expresados en unidades de $\mathrm{N}, \mathrm{P}_{2} \mathrm{O}_{5}$ y $\mathrm{K}_{2} \mathrm{O}$ por tonelada de grano producido, los valores resultantes fueron semejante a los reportados en literatura (Xu et al., 2010; $\mathrm{Hu}$ et al., 2010; Setiyono et al., 2010; Delgado et al., 2004).

La contribución de cada una de las partes de la planta de maíz a la materia seca total y a la extracción de nutrientes puede apreciarse en la Tabla 4. La espiga o panoja (inflorescencia masculina) representa aproximadamente $1.54 \%$ del peso total de la planta; las hojas $14.9 \%$; los tallos $16.9 \%$; las brácteas que rodean la mazorca $7.5 \%$; la tuza $8.2 \%$, y el grano 51 $\%$. Estos datos confirman lo estimado por $\mathrm{Hu}$ et al. (2010), respecto a las concentraciones de $\mathrm{N}$ en cada una de las partes de la planta: grano $(1.54 \%)>$ panoja $(1.48 \%)>$ hojas $(1.37 \%)>$ tuza $(0.78 \%)>$ brácteas $(0.53 \%)>$ tallo $(0.61 \%)$. Para el $\mathrm{P}$ sigue la siguiente comparación: grano $(0.42 \%)>$ panoja $(0.29 \%)>$ hojas $(0.13 \%)>$ tuza $(0.12 \%)>$ brácteas y tallos $(0.11 \%)$. Para el potasio: tallo $(2.51 \%)>$ hojas $(1.19 \%)>$ brácteas $(0.97 \%)>$ panoja $(0.93 \%)>$ tuza $(0.91 \%)>$ grano $(0.49 \%)$.
En cuanto a las extracciones totales (Tabla 4) de N $-\mathrm{P}_{2} \mathrm{O}_{5}-\mathrm{K}_{2} \mathrm{O}$, tanto en cantidades totales por hectárea, como las extracciones unitarias de los mismos por tonelada de grano, también se confirma lo encontrado por los anteriores autores, esto es, en promedio $208 \mathrm{~kg}$ de $\mathrm{N}$ por hectárea, $46.6 \mathrm{~kg}$ de $\mathrm{P}_{2} \mathrm{O}_{5}$ por hectárea, y $172.1 \mathrm{~kg}$ de $\mathrm{K}_{2} \mathrm{O}$ por hectárea (Tabla 4). Respecto a las extracciones de $\mathrm{N}-\mathrm{P}_{2} \mathrm{O}_{5}-\mathrm{K}_{2} \mathrm{O}$ en $\mathrm{kg}$ por tonelada de grano (Tabla 3), estos valores son en promedio $23.8 \mathrm{~kg}$ de $\mathrm{N}, 12.4 \mathrm{~kg}$ de $\mathrm{P}_{2} \mathrm{O}_{5}$, y $23.9 \mathrm{~kg}$ de $\mathrm{K}_{2} \mathrm{O}$. Valores similares a los encontrados por otros autores (Vásquez et al., 2014; Arrieche \& Mora, 2005; Yanez et al., 2010; Setiyono et al., 2010; Xu et al., 2013). Los porcentajes de nitrógeno, fósforo y potasio que fueron removido por el grano fueron calculados en $64.6 \%, 78.4 \%$ y $24.8 \%$, respectivamente.

\section{Conclusiones y recomendaciones.}

La extracción de nutrientes mostró la misma tendencia que los rendimientos totales (Tablas 2 y 3 ). $\mathrm{Al}$ ser expresados en unidades de $\mathrm{N}, \mathrm{P}_{2} \mathrm{O}_{5}$ y $\mathrm{K}_{2} \mathrm{O}$ por tonelada de grano producido.

Bajo las condiciones ambientales y edáficas en las que fue conducido el presente experimento, la HCP mostró la misma acción fertilizante que la urea. La aplicación del RHP, tiene muy bajo impacto a los niveles utilizados $\left(50 \mathrm{~L} \mathrm{ha}^{-1}\right)$.

En cuanto a la concentración de N, los mayores valores se dan en el grano (1.54\%) y la panoja (1.48 $\%$, la misma tendencia para la concentración de $\mathrm{P}$ (0.44\% para grano y $0.29 \%$ panoja). En cambio en $\mathrm{K}$, la mayor concentración se da en el tallo (2.51\%) y las hojas $(1.19 \%)$, siendo la menor concentración en el grano $(0.49 \%)$.

La extracción unitaria de $\mathrm{N}-\mathrm{P}_{2} \mathrm{O}_{5}-\mathrm{K}_{2} \mathrm{O}$ por tonelada de grano en promedio para todos los tratamientos del experimento fue de $23.8 \mathrm{~kg}$ en $\mathrm{N}$, $12.4 \mathrm{~kg}$ para $\mathrm{P}_{2} \mathrm{O}_{5} \mathrm{y}, 23.9 \mathrm{~kg}$ para $\mathrm{K}_{2} \mathrm{O}$.

$\mathrm{El}$ nitrógeno $\mathrm{y}$ el fósforo son principalmente removidos por el grano, mientras que el potasio es removido por las partes vegetativas de la planta.

Se recomienda realizar otros estudios con residuos hidrolizados y se debe incluir la extracción de nutrientes de las raíces.

Tabla 4. Concentración y extracción promedios en las partes de la planta, en diferentes sistemas de abonamiento, San Martín (Cañete), para biomasa de una hectárea.

\begin{tabular}{|c|c|c|c|c|c|c|c|c|}
\hline \multirow{2}{*}{$\begin{array}{l}\text { Parte de } \\
\text { planta }\end{array}$} & \multirow{2}{*}{$\begin{array}{c}\text { Biomasa } \\
\text { total }(\mathrm{kg} / \mathrm{ha})\end{array}$} & \multirow{2}{*}{$\begin{array}{c}\% \\
\text { total }\end{array}$} & \multicolumn{3}{|c|}{ Concentración (\%) } & \multicolumn{3}{|c|}{ Extracción kg ha $^{-1}$} \\
\hline & & & $\% \mathrm{~N}$ & $\% \mathbf{P}$ & $\% \mathrm{~K}$ & $\mathbf{N}$ & $\mathbf{P}$ & $\mathbf{K}$ \\
\hline Panoja & 260.60 & 1.54 & 1.48 & 0.29 & 0.93 & 3.85 & 0.76 & 2.41 \\
\hline Hojas & 2528.62 & 14.92 & 1.37 & 0.13 & 1.19 & 34.60 & 3.20 & 30.01 \\
\hline Tallos & 2571.85 & 16.90 & 0.61 & 0.11 & 2.51 & 17.37 & 3.02 & 71.94 \\
\hline Brácteas & 1280.12 & 7.47 & 0.53 & 0.11 & 0.97 & 6.83 & 1.37 & 12.37 \\
\hline Tuza & 1399.00 & 8.19 & 0.78 & 0.12 & 0.91 & 10.84 & 1.68 & 12.73 \\
\hline Grano & 8742.46 & 50.98 & 1.54 & 0.42 & 0.49 & 134.49 & 36.57 & 42.69 \\
\hline Total $\left(\mathrm{kg} \mathrm{ha}^{-1}\right)$ & 17083 & 100 & & & & 207.98 & 46.60 & 172.16 \\
\hline $\mathrm{kg} \mathrm{t}^{-1}$ grano & & & & & & 23.79 & 5.33 & 19.69 \\
\hline $\mathrm{En} \mathrm{N}-\mathrm{P}_{2} \mathrm{O}_{5}-\mathrm{K}_{2} \mathrm{O}$ & & & & & & 23.8 & 12.3 & 23.6 \\
\hline$\%$ (grano/biomasa) & & & & & & 64.6 & 78.4 & 24.8 \\
\hline
\end{tabular}




\section{Literatura citada.}

Arrieche I. \& Mora O. 2005. Efecto de la aplicación de residuos orgánicos sobre el cultivo del maíz en suelos degradados del Estado Yaracuy, Venezuela. Bioagro 17(3): $155-159$.

Bossio F. 2007. Obtención de un biofertilizante basado en residuos de pescado y roca fosfatada. Tesis Biólogo UNALM. Lima, Perú, 62 p.

Cayuela M., Mondini C., Insma H., Sinicco T. \& FrankeWhittle I. 2009. Plant and animal wastes composting: Effects of the $\mathrm{N}$ source on process performance. Bioresource Technology 100: 3097-3106.

Comese R., Gonzáles M. \& Conti, M. 2009. Cambios en las propiedades de suelo de huerta y rendimiento de Beta vulgaris var. Cycla (L) por el uso de enmiendas orgánicas. Ciencia del Suelo 27(2): 271-275.

Delgado R., Núñez M. \& Velásquez L. 2004. Acumulación de materia seca, absorción de nitrógeno, fósforo $\mathrm{y}$ potasio por el maíz en diferentes condiciones de manejo de la fertilización nitrogenada. Agronomía Tropical 54(4): $372-389$

Documet L. 2015. Obtención de harina de cuernos y pezuñas de ganado bovino (Bos taurus) y evaluación de su aplicación como abono orgánico. Tesis Magister Scientiae en Ciencias Ambientales. Escuela de Posgrado. Universidad Nacional Agraria La Molina. (Publicación en prensa).

Garzón I. 2010. Diagnóstico ambiental del camal municipal de la ciudad de Santo Domingo y mejora de su gestión. Proyecto para la obtención del Título de Ingeniero Ambiental. Escuela Politécnica Nacional. Quito, Ecuador. $115 \mathrm{p}$.

Hu H., Bai Y., Yang L., Kong Q., Lu Y., Wang L. \& Wang Z. 2010. Response of element distribution of various organs of maize to fertilizer application. Agricultural Sciences in China 9(3): 401-407.

Jones, J.B.J. 1991. Kjeldahl method for nitrogen determination. Athens, GA: Micro-Macro Publishing Inc.

Korniłłowicz T. \& Bohacz J. 2011. Biodegradation of keratin waste: Theory and practical aspects. Waste Management. 31 1689-1701. DOI:10.1016/j.wasman.2011.03.024.

Knuckey I., Sinclair C., Surapaneni A. \& Ashcroft W. 2004. Utilization of seafood processing waste. Challenges and opportunities. En: $3^{\text {rd }}$ Australian New Zealand Soils Conference.

López M.E. \& Sainz M. J. 2011. Gestión de residuos orgánicos de uso agrícola. Santiago de Compostela: Servizo de Publicacións e Intercambio Científico, Universidade de Santiago de Compostela. 226 p.

Ospina J., Vanegas H. \& Polanía F. 2011. Evaluación de la producción de biomasa de maíz en condiciones del trópico colombiano. Federación Nacional de Cultivadores de Cereales y Leguminosas -FENALCE. Disponible http://www.fenalce.org/arch_public/biomasa98.pdf
Prystupa P., Salvagiotti F., Ferraris G., Gutierrez F., Elisei J. \& Couretod L. 2004. Efecto de la fertilización con fósforo, azufre y potasio en cultivo de maíz en la pampa ondulada. Informaciones Agronómicas 23.

Rustad T. 2003. Utilisation of marine by-products. Electronic Journal of Environmental, Agricultural and Food Chemistry 2(4): 458-463.

Seleiman M., Santanen A., Jaakola S., Ekholm P., Hartikainen H., Stoddart F. \& Mäkelä P. 2013. Biomass yield and quality of bioenergy crops grown with synthetic and organic fertilizers. Biomass and Bioenergy 59: 477-485.

Serna C., Trujillo L. \& Urrea R. 2011. Respuesta del maíz (Zea mays L.) a la aplicación edáfica de N-P-K en un andisol de la región centro-occidente de Caldas. Agronomía 19: 68-76.

Setiyono T.D., Walters D.T., Cassman K.G., Witt C. \& Dobermann A. 2010. Estimating maize nutrient uptake requirements. Field Crops Research 118(2): 158-168.

Silva W., Alfaro Y. \& Jiménez R. 2009. Evaluación de las características morfológicas y agronómicas de cinco líneas de maíz amarillo en diferentes fechas de siembra. Revista Científica UDO Agrícola 9(4): 743-755.

Vásquez A., Zetina, R. \& Meneses I. 2014. Extracciones nutrimentales en tres cultivos en Veracruz, México. Revista Científica Biológico Agropecuaria Tuxpan 2(3): 347-353.

Vincenza F., Cruz I., Ferreira R., Malcata F.X., Pintado M. \& Castro P. 2010. Valorisation of natural extracts from marine source focused on marine by-products: A review. Disponible http://repositorio.ucp.pt/bitstream/10400.14/4897/3/FRI N32751\%201\%20\%282\%29.pdf

Windsor M. \& Barlow S. 1984 Introducción a los subproductos de pesquería. Zaragoza, España. Acribia. $204 \mathrm{p}$.

Xia Y., Wang D., Kong Y., Ungerfeld E., Seviour R. \& Massé D. 2015. Anaerobic digestibility of beef hooves with swine manure or slaughterhouse sludge. Waste Management 38: 443-448.

Xu X., He P., Pampolino M., Chuan L., Johnston, A. Qiu, S., Zhao, S. \& Zhou, W. 2013. Nutrient requirements for maize in China based on QUEFTS analysis. Field Crops Research 150: 115-125.

Yanez D., Valverde F. \& Cartagena Y. 2010. Evaluación del elemento faltante en el cultivo de maíz (Zea mays L.) en la Provincia Bolívar. XII Congreso Ecuatoriano de la Ciencia del Suelo.17-19 noviembre de 2010. Santo Domingo- Ecuador.

Zasoski R.J. \& Burau R G. 1977. A rapid nitric-perchloric acid digestion method for multi-element tissue analysis. Communications in Soil Science and Plant Analysis 8: 425-436.

Zoccola M., Aluigi, A. \& Tonin, C. 2009. Characterisation of keratin biomass from butchery and wool industry wastes. Journal of Molecular Structure 938(1-3): 35-40.

\footnotetext{
${ }^{1}$ Departamento Académico de Suelos. Facultad de Agronomía. Universidad Nacional Agraria La Molina. Av.

La Molina S/N, La Molina Lima Perú. gaguirre@lamolina.edu.pe jalegre@lamolina.edu.pe
} 